\title{
NIJMEGEN SOFT-CORE POTENTIAL INCLUDING TWO-MESON EXCHANGE
}

\author{
V.G.J. Stoks \\ The Flinders University of South Australia, \\ Bedford Park, South Australia 5042, Australia \\ Th.A. Rijken \\ Institute for Theoretical Physics, University of Nijmegen, \\ Nijmegen, The Netherlands
}

\begin{abstract}
We report on the progress of the construction of the extended soft-core (ESC) Nijmegen potential. Next to the standard one-boson-exchange parts, the model includes the pion-meson-exchange potentials due to the parallel and crossed-box diagrams, as well as the one-pair and two-pair diagrams, vertices for which can be identified with similar interactions appearing in chiralsymmetric Lagrangians. Although the ESC potential is still under construction, it already gives an excellent description of all $N N$ scattering data below $350 \mathrm{MeV}$ with $\chi^{2} /$ datum $=1.3$.
\end{abstract}

\section{INTRODUCTION}

About two years ago [1], the Nijmegen group investigated the quality with respect to the $p p$ scattering data below $350 \mathrm{MeV}$ of a number of $N N$ potential models. We found that only a few of the models we investigated were of satisfactory quality. But even the best models still gave $\chi^{2} /$ datum $\approx 2$. To be specific, only potentials which are explicitly fitted to the $p p$ data give a reasonable description of these data. Models which have been fitted only to the $n p$ data (including the most recent $n p$ potentials) in general give a very poor description of the $p p$ data. (Obviously, before confronting an $n p$ potential with the $p p$ data, one has to make the proper electromagnetic corrections and account for the fact that its parameters were fitted to the $n p{ }^{1} S_{0}$ scattering length). Another problem is that at the time the older potential models were constructed, the $n p$ data were too inaccurate to really sufficiently constrain the fit to the isoscalar partial waves. Over the last decade, however, the quality and accuracy of the $n p$ data has improved considerably. So if these older models are now confronted with the presently available $n p$ data, they do a very poor job indeed.

The $\chi^{2} /$ datum $\approx 2$ of the best potential models (and then usually either only for the $p p$ data or the $n p$ data) should be compared with the quality of the Nijmegen partial-wave analysis [2] where we reached $\chi^{2} /$ datum $=1$ for 
both $p p$ and $n p$. This is about the best one can get.

Now that we have finished our partial-wave analyses of the $p p$ and $n p$ scattering data below $350 \mathrm{MeV}$, we have access to a carefully scrutinized database, which can be used to try to construct a potential model which does give an excellent description of the $p p$ and $n p$ scattering data. As a first attempt to construct such a high-quality potential the parameters of the old Nijm78 NN potential [3] were refitted. However, even with some modifications (inclusion of the mass difference between neutral and charged pions) and some additional parameters (different cutoff parameters for each type of meson exchange), this new Nijm93 potential [4] is only partially successful in that we still couldn't get lower than $\chi^{2} /$ datum $=1.9$. On the other hand, the Nijm93 potential provides an enormous improvement over the old Nijm78 potential in its fit to the $n p$ data. Taking into consideration the fact that other one-boson-exchange $(\mathrm{OBE})$ potentials also cannot do much better than $\chi^{2} /$ datum $\approx 2$, one can conclude that apparently the OBE model lacks some important physics.

Amongst other things, this motivated us to go beyond the OBE approach by including the two-meson-exchange contributions as well. The first results of this extended soft-core (ESC) model were already reported at the European Few-Body Conference [5]. The advantage of this extension of the OBE model is that the coupling constants are subject to physical interpretation. Hence, it is possible to extend this model even further and use SU(3) symmetry to construct a general baryon-baryon potential with only a minimum of new free parameters. This is what we intend to do in the near future.

A possible alternative approach to arrive at a high-quality potential is to resort to the construction of purely phenomenological potential models. Introducing a sufficient number of free parameters and spending enough effort in fitting the model to the $N N$ data, it should in principle be easy to construct a model with $\chi^{2} /$ datum $\approx 1$. The Nijmegen group has constructed several of these models [4]. Also, one of us is involved in the construction of a new updated version of the phenomenological Argonne potential [6]. However, one can argue that these models are only relevant in the $N N$ sector, since it doesn't make any sense, for example, to apply $\mathrm{SU}(3)$ to the coupling constants in order to construct a $Y N$ potential.

\section{EXTENDED SOFT-CORE POTENTIAL}

The OBE part of the ESC model is constructed in the standard way from the scalar, pseudovector, and vector nucleon-nucleon-meson Lagrangians

$$
\begin{aligned}
\text { scalar } & : \quad \mathcal{L}\left(0^{++}\right)=g_{S} \bar{\psi} \psi \phi \\
\text { pseudovector } & : \quad \mathcal{L}\left(0^{-+}\right)=\frac{f_{P}}{m_{\pi}} \bar{\psi} \gamma_{5} \gamma_{\mu} \psi \partial^{\mu} \phi
\end{aligned}
$$




$$
\text { vector } \quad: \quad \mathcal{L}\left(1^{--}\right)=g_{V} \bar{\psi} \gamma_{\mu} \psi \phi^{\mu}+\frac{f_{V}}{4 \mathcal{M}} \bar{\psi} \sigma_{\mu \nu} \psi\left(\partial^{\mu} \phi^{\nu}-\partial^{\nu} \phi^{\mu}\right)
$$

We include all non-strange mesons $\left(a_{0}, \epsilon, f_{0}\right),\left(\pi, \eta, \eta^{\prime}\right)$, and $(\rho, \omega, \phi)$, as well as the dominant $J=0$ parts of the Pomeron, and of the $\left(a_{2}, f_{2}, f_{2}^{\prime}\right)$ tensor-meson trajectories. The latter give rise to Gaussian potentials, and are a typical feature of the Nijmegen soft-core potentials. For the two-meson-exchange potentials we calculate the so-called Brueckner-Watson parallel and crossed-box diagrams with Gaussian form factors [7]. Next to $\pi-\pi$ exchange we also include the other lowest-mass $\pi$-meson exchanges, viz. $\pi-\epsilon, \pi-\eta, \pi-\rho$, and $\pi-\omega$. A motivation for the inclusion of these interactions is that they are also important for a proper low-energy description of the pion-nucleon $\rightarrow$ meson-nucleon amplitude. Furthermore, because of the small pion mass, the range of the pion-meson potentials is comparable to the range of the meson-exchange potentials.

We also include the one-pair and two-pair contributions to the two-meson potentials. They can be viewed upon as the result of the 'out-integration' of the heavy-meson and resonance degrees of freedom. Another motivation for including these 'pair' vertices is that similar interactions appear in chiralsymmetric Lagrangians. We consider the following nucleon-nucleon-mesonmeson Lagrangians:

$$
\begin{aligned}
\text { scalar }: & \mathcal{L}\left(0^{++}\right)=\bar{\psi} \psi\left[g_{(\pi \pi)_{0}} \boldsymbol{\pi} \cdot \boldsymbol{\pi}+g_{(\sigma \sigma)} \sigma^{2}\right] / m_{\pi}, \\
\text { vector }: & \mathcal{L}\left(1^{--}\right)=\left[g_{(\pi \pi)_{1}} \bar{\psi} \gamma_{\mu} \boldsymbol{\tau} \psi-\frac{f_{(\pi \pi)_{1}}}{2 \mathcal{M}} \bar{\psi} \sigma_{\mu \nu} \boldsymbol{\tau} \psi \partial^{\nu}\right] \cdot\left(\boldsymbol{\pi} \times \partial^{\mu} \boldsymbol{\pi}\right) / m_{\pi}^{2}, \\
\operatorname{axial}(1): & \mathcal{L}\left(1^{++}\right)=g_{(\pi \rho)_{1}} \bar{\psi} \gamma_{5} \gamma_{\mu} \boldsymbol{\tau} \psi \cdot\left(\boldsymbol{\pi} \times \boldsymbol{\rho}^{\mu}\right) / m_{\pi}, \\
& \text { and also } g_{(\pi \sigma)} \bar{\psi} \gamma_{5} \gamma_{\mu} \boldsymbol{\tau} \psi \cdot\left(\sigma \partial^{\mu} \boldsymbol{\pi}-\boldsymbol{\pi} \partial^{\mu} \sigma\right) / m_{\pi}^{2}, \\
\operatorname{axial}(2): \quad & \mathcal{L}\left(1^{+-}\right)=-i g_{(\pi \rho)_{0}} \bar{\psi} \gamma_{5} \sigma_{\mu \nu} \psi \partial^{\nu}\left(\boldsymbol{\pi} \cdot \boldsymbol{\rho}^{\mu}\right) / m_{\pi}^{2}, \\
& \text { and also }-i g_{(\pi \omega)} \bar{\psi} \gamma_{5} \sigma_{\mu \nu} \boldsymbol{\tau} \psi \cdot \partial^{\nu}\left(\boldsymbol{\pi} \omega^{\mu}\right) / m_{\pi}^{2},
\end{aligned}
$$

where $\sigma$ is to be identified with the broad $\epsilon$ scalar meson. Retaining only the leading-order terms of the vertices and following Ref. [7], it is straightforward to work out the resulting one-pair and two-pair two-meson-exchange potentials in momentum space [8]. As in the Nijmegen OBE model, we attach a Gaussian form factor $\exp \left(-\mathbf{k}^{2} / 2 \Lambda^{2}\right)$ to each vertex, where now the cutoff mass $\Lambda$ depends on the type of meson (scalar, pseudovector, or vector) being exchanged. This still allows the momentum-space potentials to be Fourier transformed to coordinate space, be it at the expense of a one-dimensional integral in some of the potentials. Details can be found in Refs. $[7,8]$.

\section{RESULTS AND DISCUSSION}

Taking the Nijmegen OBE potential as a starting point, we include the twomeson potentials and refit the coupling constants and cutoffs. The parameters 
are fitted to the $\chi^{2}$ hypersurface of the recently finished Nijmegen partial-wave analysis [2] of the $p p$ and $n p$ scattering data below $350 \mathrm{MeV}$. The database contains $1787 p p$ data and $2514 n p$ data and, although the fit is still constantly being improved upon, at present we can quote the already very promising result of $\chi^{2} /$ datum $=1.3$.

The pion-nucleon coupling constant was searched for and the value at the pion pole of $f^{2}=0.072$, is somewhat lower than our determination in the $N N$ partial-wave analysis [9]. The $(f / g)_{\rho}$ ratio is 4.0 which is very close to the vector-meson dominance value of 3.7 , but the present value $g_{\rho} \approx 1.0$ is still a bit high. The $g_{(\pi \rho)_{1}}$ and $g_{(\pi \sigma)}$ coupling constants are close to the values expected from $A_{1}$ dominance (see [5]). The $p p$ and $n p$ phase shifts are all very close to the values as determined in our partial-wave analysis [2], which is to be expected with such a low $\chi^{2}$. Especially the results for the ${ }^{1} P_{1}$ and ${ }^{3} D_{2}$ phase shifts are a major improvement over the values of the old Nijm78 potential. Because we take average nucleon masses and average pion masses in the twomeson potentials, the difference between the $p p$ and $n p{ }^{1} S_{0}$ potentials is too small to account for the difference between the $p p$ and $n p$ scattering lengths. As we did in the Nijm93 potential [4], we therefore still have to introduce a phenomenological parameter to be able to fit both scattering lengths.

To summarize, the results for the ESC potential look very promising. The advantage of this $N N$ potential is that we can try to use $\mathrm{SU}(3)$ symmetry in order to extend it to a general baryon-baryon potential. This would only require the introduction of a minimal set of new free parameters; e.g., coupling constants for strange mesons coupling to nucleons and/or hyperons. Clearly, this is an important advantage over purely phenomenological $N N$ potentials.

\section{REFERENCES}

1. V. Stoks and J.J. de Swart, Phys. Rev. C 47, 761 (1993).

2. V.G.J. Stoks, R.A.M. Klomp, M.C.M. Rentmeester, and J.J. de Swart, Phys. Rev. C 48, 792 (1993).

3. M.M. Nagels, T.A. Rijken, and J.J. de Swart, Phys. Rev. D 17, 768 (1978).

4. V.G.J. Stoks, R.A.M. Klomp, C.P.F. Terheggen, and J.J. de Swart, submitted to Phys. Rev. C.

5. Th.A. Rijken, Baryon-Baryon Interactions, Proceedings of the XIVth European Conference on Few-Body Problems in Physics, ed. B.L.G. Bakker and R. van Dantzig, Amsterdam, The Netherlands, 1993.

6. R.B. Wiringa, R.A. Smith, and T.L. Ainsworth, Phys. Rev. C 29, 1207 (1984); R.B. Wiringa, V.G.J. Stoks, and R. Schiavilla, in preparation.

7. Th.A. Rijken, Ann. Phys. (NY) 208, 253 (1991); Th.A. Rijken and V.G.J. Stoks, in preparation.

8. Th.A. Rijken and V.G.J. Stoks, in preparation.

9. V. Stoks, R. Timmermans, and J.J. de Swart, Phys. Rev. C 47, 512 (1993). 